\title{
A Novel Shape-Free Plane Quadratic Polygonal Hybrid Stress-Function Element
}

\author{
Pei-Lei Zhou' ${ }^{1}$ and Song Cen ${ }^{1,2,3}$ \\ ${ }^{1}$ Department of Engineering Mechanics, School of Aerospace Engineering, Tsinghua University, Beijing 100084, China \\ ${ }^{2}$ High Performance Computing Center, School of Aerospace Engineering, Tsinghua University, Beijing 100084, China \\ ${ }^{3}$ Key Laboratory of Applied Mechanics, School of Aerospace Engineering, Tsinghua University, Beijing 100084, China \\ Correspondence should be addressed to Song Cen; censong@tsinghua.edu.cn
}

Received 29 August 2014; Accepted 3 September 2014

Academic Editor: Zhiqiang $\mathrm{Hu}$

Copyright (c) 2015 P.-L. Zhou and S. Cen. This is an open access article distributed under the Creative Commons Attribution License, which permits unrestricted use, distribution, and reproduction in any medium, provided the original work is properly cited.

\begin{abstract}
A novel plane quadratic shape-free hybrid stress-function (HS-F) polygonal element is developed by employing the principle of minimum complementary energy and the fundamental analytical solutions of the Airy stress function. Without construction of displacement interpolation function, the formulations of the new model are much simpler than those of the displacement-based polygonal elements and can be degenerated into triangular or quadrilateral elements directly. In particular, it is quite insensitive to various mesh distortions and even can keep precision when element shape is concave. Furthermore, the element does not show any spurious zero energy modes. Numerical examples show the excellent performance of the new element, denoted by HSF-AP-19 $\beta$, in both displacement and stress solutions.
\end{abstract}

\section{Introduction}

The conventional triangular and quadrilateral elements are widely used in 2D finite element analyses $[1,2]$. However, when simulating microstructure problems, inconveniences may still exist. For instance, the shape of one crystal of plane polycrystalline materials is usually regarded as a polygon. If these microstructures are meshed by those conventional triangular and quadrilateral elements, the density of the elements will be extremely high. But if each crystal is modeled only by one polygonal element, the related computation costs can be dramatically reduced [3]. Actually, the polygonal elements are more flexible for meshing structures with complex shapes [4-6] or connecting different kinds of meshes $[7,8]$, so that they possess obvious advantages for simulating heterogeneous materials [9-13].

Compared with the conventional finite elements, it is more difficult to formulate the interpolation functions for polygonal elements because of the arbitrariness of the element shape. Many researchers have made great efforts on developing effective techniques to solve this problem. In 1975, Wachspress [14] firstly suggested to take the rational functions as the element shape functions. These functions satisfy Kronecker-delta property and continuity requirement along the polygon and can keep linear on the adjacent edges and zero on the opposite edge. However, their construction procedures were relatively complicated. Later, a series of improvements on the Wachspress interpolation were successfully proposed [2, 15-23]. Beside the Wachspress method, the Laplace functions were also utilized to build the interpolation functions for polygonal elements, such as the works proposed by Wang and Li [24] and Sukumar and Tabarraei [25]. Another mentionable approach is the barycentric coordinate method [26-28] which can be applied to develop both conventional and polygonal elements. During recent years, new polygonal elements are still emerging in many literatures. Dohrmann et al. [7] developed a transition element for connecting dissimilar finite element meshes; Peters and Heymsfield [29] developed constant strain elements consisting of an arbitrary number of nodes; Liu et al. [30, 31] constructed several linear 
polygonal elements by the smoothed finite element method; Chen et al. [32] proposed a quadratic spline element based on area coordinates and B-net method; Sukumar [33] proposed an arbitrary quadratic polygonal element by applying quadratic maximum-entropy serendipity shape functions. All the elements mentioned above belong to displacementbased elements, in which the displacement interpolation functions satisfying compatibility requirements must be considered.

In order to avoid the difficulties in constructions of displacement-based polygonal elements, some researchers also utilized the hybrid finite element method to develop polygonal elements. Based on the principle of minimum complementary energy, Ghosh and Mallett [34] proposed the Voronoi cell finite element method (VCFEM), in which no displacement interpolation function is needed. By applying the Hellinger-Reissner variational principle, Zhang and Katsube $[35,36]$ developed the hybrid polygonal element (HPE) to simulate materials with inclusions and holes. Peng et al. $[37,38]$ proposed a novel base force element method by using the principle of minimum complementary energy and developed quadratic polygonal elements to solve problems with concave polygonal meshes.

Recently, Cen et al. [39-44] proposed a hybrid stressfunction (HS-F) element method based on the principle of minimum complementary energy and the fundamental analytical solutions of Airy stress function. They constructed several elements with excellent performance, such as 8node and 12-node plane quadrilateral elements and 4node plane quadrilateral element with drilling degrees of freedom. These quadrilateral elements are not sensitive to severely distorted meshes and can work well even when the element shapes degenerate into concave quadrilaterals or triangles. So, they are called shape-free finite elements since their performances are independent to the element shapes. Furthermore, this HS-F method can be directly and easily extended to develop arbitrary polygonal element due to its flexible theoretical frame. In the paper, a quadratic polygonal element will be developed by the above HS-F approach.

The arrangements of this paper are as follows. In Section 2, the 2D HS-F element method is briefly reviewed. Then, a new quadratic polygonal HS-F element, denoted by HSF-AP-19 $\beta$, is formulated in Section 3. Several standard numerical examples are performed in Section 4 to validate the high performance of the new element. Finally, some conclusion remarks are given in Section 5.

\section{Brief Reviews on the Plane HS-F Element Method}

For a $2 \mathrm{D}$ finite element model, the complementary energy functional can be written as [39-44]

$$
\Pi_{C}=\frac{1}{2} \iint_{A^{e}} \boldsymbol{\sigma}^{\mathrm{T}} \mathbf{C} \boldsymbol{\sigma} t \mathrm{~d} A-\int_{\Gamma^{e}} \mathrm{~T}^{\mathrm{T}} \overline{\mathbf{u}} t \mathrm{~d} s,
$$

with

$$
\begin{gathered}
\boldsymbol{\sigma}=\left\{\begin{array}{c}
\sigma_{x} \\
\sigma_{y} \\
\tau_{x y}
\end{array}\right\}, \quad \mathbf{T}=\left\{\begin{array}{l}
T_{x} \\
T_{y}
\end{array}\right\}=\left[\begin{array}{lll}
l & 0 & m \\
0 & m & l
\end{array}\right]\left\{\begin{array}{c}
\sigma_{x} \\
\sigma_{y} \\
\tau_{x y}
\end{array}\right\}=\mathbf{L} \boldsymbol{\sigma}, \\
\overline{\mathbf{u}}=\left\{\begin{array}{l}
\bar{u} \\
\bar{v}
\end{array}\right\},
\end{gathered}
$$

in which $t$ is the thickness of the element; $A^{e}$ is the element area; $\Gamma^{e}$ is the element boundary; $\boldsymbol{\sigma}$ is the element stress vector; $\mathbf{T}$ is the traction force vector along the element boundaries; $l$ and $m$ are the direction cosines of the outer normal $\vec{n}$ of the element boundaries; $\mathbf{C}$ is the elasticity matrix of compliances, and, for isotropic cases, it can be expressed by

$$
\begin{aligned}
& \mathrm{C}=\frac{1}{E^{\prime}}\left[\begin{array}{ccc}
1 & -\mu^{\prime} & 0 \\
-\mu^{\prime} & 1 & 0 \\
0 & 0 & 2\left(1+\mu^{\prime}\right)
\end{array}\right], \\
& \text { plane stress: } E^{\prime}=E, \quad \mu^{\prime}=\mu, \\
& \text { plane strain: } E^{\prime}=\frac{E}{\left(1-\mu^{2}\right)}, \quad \mu^{\prime}=\frac{\mu}{(1-\mu)}, \\
& E \text {, Young's modulus; } \mu \text {, Poisson's ratio. }
\end{aligned}
$$

$\overline{\mathbf{u}}$ is the assumed displacement vector along element boundaries and can be interpolated by the element nodal displacement vector $\mathbf{q}^{e}$ :

$$
\overline{\mathbf{u}}=\left\{\begin{array}{l}
\bar{u} \\
\bar{v}
\end{array}\right\}=\left.\overline{\mathbf{N}}\right|_{\Gamma} \mathbf{q}^{e},
$$

where $\left.\overline{\mathbf{N}}\right|_{\Gamma}$ is the interpolation function matrix for element boundary displacements.

The element stress fields are assumed as follows:

$$
\begin{gathered}
\boldsymbol{\sigma}=\boldsymbol{\sigma}^{0}+\boldsymbol{\sigma}^{*}=\left\{\begin{array}{c}
\sigma_{x}^{0} \\
\sigma_{y}^{0} \\
\tau_{x y}^{0}
\end{array}\right\}+\left\{\begin{array}{c}
\sigma_{x}^{*} \\
\sigma_{y}^{*} \\
\tau_{x y}^{*}
\end{array}\right\}=\mathbf{S} \boldsymbol{\beta}+\boldsymbol{\sigma}^{*}, \\
\boldsymbol{\beta}=\left[\begin{array}{llllll}
\beta_{1} & \beta_{2} & \beta_{3} & \cdots & \cdots & \beta_{N}
\end{array}\right]^{\mathrm{T}},
\end{gathered}
$$

where $\beta_{i}(i=1 \sim N)$ are $N$ unknown stress parameters; $\mathbf{S}$ is the stress solution matrix:

$$
\mathbf{S}=\left[\begin{array}{ccccc}
\sigma_{x 1} & \sigma_{x 2} & \sigma_{x 3} & \cdots & \sigma_{x k} \\
\sigma_{y 1} & \sigma_{y 2} & \sigma_{y 3} & \cdots & \sigma_{y k} \\
\tau_{x y 1} & \tau_{x y 2} & \tau_{x y 3} & \cdots & \tau_{x y k}
\end{array}\right]_{3 \times k}
$$

The components (stress interpolation functions) in $\mathbf{S}$ are all derived from $k$ fundamental solutions of stress function $\phi$. The first nineteen solutions are given in Table 1. 
TABLE 1: Nineteen fundamental analytical solutions of the Airy stress function and resulting stress solutions for plane problem.

(a)

\begin{tabular}{lcccccccccc}
\hline$i$ & 1 & 2 & 3 & 4 & 5 & 6 & 7 & 8 & 9 & 10 \\
\hline$\phi_{i}$ & $x^{2}$ & $x y$ & $y^{2}$ & $x^{3}$ & $x^{2} y$ & $x y^{2}$ & $y^{3}$ & $x^{3} y$ & $x y^{3}$ & $x^{4}-y^{4}$ \\
\hline$\sigma_{x i}$ & 0 & 0 & 2 & 0 & 0 & $2 x$ & $6 y$ & 0 & $6 x y$ & $-12 y^{2}$ \\
$\sigma_{y i}$ & 2 & 0 & 0 & $6 x$ & $2 y$ & 0 & 0 & $6 x y$ & 0 & $12 x^{2}$ \\
$\tau_{x y i}$ & 0 & -1 & 0 & 0 & $-2 x$ & $-2 y$ & 0 & $-3 x^{2}$ & $-3 y^{2}$ & 0 \\
\hline
\end{tabular}

(b)

\begin{tabular}{lcccccc}
\hline$i$ & 11 & 12 & 13 & 14 & 15 & 16 \\
\hline$\phi_{i}$ & $6 x^{2} y^{2}-x^{4}-y^{4}$ & $x^{3} y^{2}-x y^{4}$ & $5 x^{3} y^{2}-x^{5}$ & $x^{2} y^{3}-x^{4} y$ & $5 x^{2} y^{3}-y^{5}$ & $x^{5} y-x y^{5}$ \\
\hline$\sigma_{x i}$ & $12\left(x^{2}-y^{2}\right)$ & $2 x\left(x^{2}-6 y^{2}\right)$ & $10 x^{3}$ & $6 x^{2} y$ & $10 y\left(3 x^{2}-2 y^{2}\right)$ & $-20 x y^{3}$ \\
$\sigma_{y i}$ & $-12\left(x^{2}-y^{2}\right)$ & $6 x y^{2}$ & $-10 x\left(2 x^{2}-3 y^{2}\right)$ & $-2 y\left(6 x^{2}-y^{2}\right)$ & $10 y^{3}$ & $20 x^{3} y$ \\
$\tau_{x y i}$ & $-24 x y$ & $-2 y\left(3 x^{2}-2 y^{2}\right)$ & $-30 x^{2} y$ & $2 x\left(2 x^{2}-3 y^{2}\right)$ & $-30 x y^{2}$ & $-5 x^{4}+5 y^{4}$ \\
\hline
\end{tabular}

(c)

\begin{tabular}{lccc}
\hline$i$ & 17 & 18 & 19 \\
\hline$\phi_{i}$ & $10 x^{3} y^{3}-3 x^{5} y-3 x y^{5}$ & $x^{6}-10 x^{4} y^{2}+5 x^{2} y^{4}$ & $y^{6}-10 x^{2} y^{4}+5 x^{4} y^{2}$ \\
\hline$\sigma_{x i}$ & $60 x^{3} y-60 x y^{3}$ & $-20 x^{4}+60 x^{2} y^{2}$ & $30 y^{4}-120 x^{2} y^{2}+10 x^{4}$ \\
$\sigma_{y i}$ & $60 x y^{3}-60 x^{3} y$ & $30 x^{4}-120 x^{2} y^{2}+10 y^{4}$ & $-20 y^{4}+60 x^{2} y^{2}$ \\
$\tau_{x y i}$ & $-90 x^{2} y^{2}+15 x^{4}+15 y^{4}$ & $80 x^{3} y-40 x y^{3}$ & $80 x y^{3}-40 x^{3} y$ \\
\hline
\end{tabular}

$\boldsymbol{\sigma}^{*}$ is a particular solution corresponding to body forces, and, for constant body forces $b_{x}$ and $b_{y}$, it can be given by

$$
\boldsymbol{\sigma}^{*}=\left\{\begin{array}{c}
\sigma_{x}^{*} \\
\sigma_{y}^{*} \\
\tau_{x y}^{*}
\end{array}\right\}=\left\{\begin{array}{c}
0 \\
0 \\
-b_{y} x-b_{x} y
\end{array}\right\} .
$$

Therefore, these components are also fundamental stress solutions which satisfy all governing equations. This is the key point for developing the hybrid stress-function (HS-F) element models. According to the principle of minimum complementary energy [39-44], we have

$$
\begin{aligned}
& \boldsymbol{\beta}=\mathbf{M}^{-1}\left(\mathbf{H q}^{e}-\mathbf{M}^{*}\right) \text { with } \mathbf{M}=\iint_{A^{e}} \mathbf{S}^{\mathrm{T}} \mathbf{C S} t \mathrm{~d} A, \\
& \mathbf{M}^{*}=\iint_{A^{e}} \mathbf{S}^{\mathrm{T}} \mathbf{C} \boldsymbol{\sigma}^{*} t \mathrm{~d} A .
\end{aligned}
$$

By applying the principle of the minimum complementary energy again, the final finite element equation of the hybrid stress-function (HS-F) element method can be written as

$$
\sum_{e} \mathbf{K}^{e} \mathbf{q}^{e}=\sum_{e} \mathbf{p}^{e}
$$

where $\mathbf{K}^{e}$ is the element stiffness matrix of $2 \mathrm{D}$ hybrid stressfunction elements:

$$
\mathbf{K}^{e}=\mathbf{H}^{\mathrm{T}} \mathbf{M}^{-1} \mathbf{H},
$$

with

$$
\mathbf{H}=\left.\int_{\Gamma^{e}} \mathbf{S}^{\mathrm{T}} \mathbf{L}^{\mathrm{T}} \overline{\mathbf{N}}\right|_{\Gamma} t \mathrm{~d} s
$$

$\mathbf{p}^{e}$ is the element equivalent load vector. Its part caused by concentrated and distributed line forces can be determined by the standard procedure for the conventional finite elements. And the part caused by body forces is given by

$$
\mathbf{p}^{e}=\mathbf{H}^{\mathrm{T}} \mathbf{M}^{-1} \mathbf{M}^{*}-\mathbf{V}^{\mathrm{T}} \text { with } \mathbf{V}=\left.\int_{\Gamma^{e}} \boldsymbol{\sigma}^{* \mathrm{~T}} \mathbf{L}^{\mathrm{T}} \overline{\mathbf{N}}\right|_{\Gamma} t \mathrm{~d} s .
$$

Once the element nodal displacement vector $\mathbf{q}^{e}$ is solved, the stresses at any point within the element can be given by

$$
\boldsymbol{\sigma}=\left\{\begin{array}{c}
\sigma_{x} \\
\sigma_{y} \\
\tau_{x y}
\end{array}\right\}=\mathbf{S M}^{-1}\left(-\mathbf{M}^{*}+\mathbf{H q}^{e}\right)+\boldsymbol{\sigma}^{*}
$$

\section{Formulations of a New Plane Quadratic Polygonal HS-F Element}

As shown in Figure 1, consider an arbitrary $n$-sided quadratic polygonal HS-F element; the element nodal displacement vector $\mathbf{q}^{e}$ is defined as

$$
\mathbf{q}^{e}=\left[\begin{array}{llllllllll}
u_{1} & v_{1} & u_{2} & v_{2} & \cdots & \cdots & u_{2 n-1} & v_{2 n-1} & u_{2 n} & v_{2 n}
\end{array}\right]^{\mathrm{T}},
$$

in which $u_{i}$ and $v_{i}(i=1-2 n)$ are the nodal displacements in $x$ - and $y$-directions, respectively.

3.1. Displacement Interpolation along the Element Boundary. The element boundary displacement vector can be described by the element nodal displacement vector (see (4)). For 


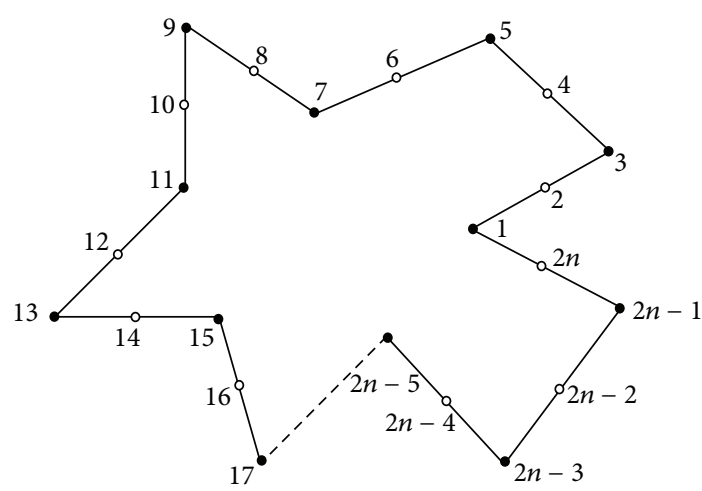

- Corner node

○ Midside node

FIGURE 1: An arbitrary n-sided polygonal HS-F element.

element edge with three nodes $i, j=i+1$, and $k=j+1$ (if $k>2 n$, let $k=1$ ), let

$$
\begin{aligned}
& x=\bar{N}_{1}(\xi) x_{i}+\bar{N}_{2}(\xi) x_{j}+\bar{N}_{3}(\xi) x_{k} \\
& y=\bar{N}_{1}(\xi) y_{i}+\bar{N}_{2}(\xi) y_{j}+\bar{N}_{3}(\xi) y_{k}
\end{aligned}
$$

with

$$
\begin{gathered}
\bar{N}_{1}=-0.5 \xi(1-\xi), \quad \bar{N}_{2}=1-\xi^{2} \\
\bar{N}_{3}=0.5 \xi(1+\xi)
\end{gathered}
$$

where $\xi$ is the local coordinate along each element boundary and $-1 \leq \xi \leq 1$.

Then, for $i$ th edge $\Gamma_{i}$, the matrix $\left.\overline{\mathbf{N}}\right|_{\Gamma}$ in (4) can be expressed by

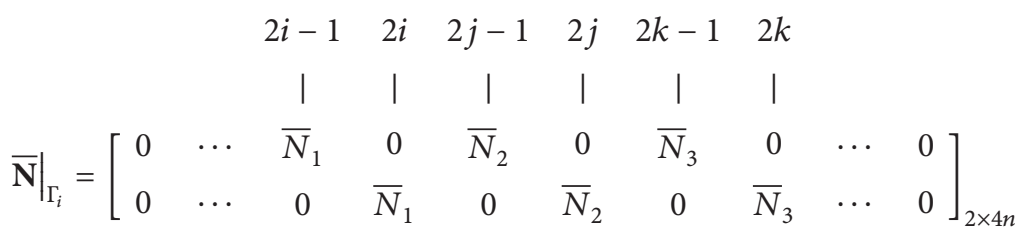

(if $k>2 n$, let $k=1$ ).

3.2. Evaluation Procedure for the Matrix $\boldsymbol{H}$ in (11). For an arbitrary $n$-sided polygonal element, the evaluation of matrix $\mathbf{H}$ in (11) should be performed along the whole element boundaries. Thus, (11) can be rewritten as

$$
\mathbf{H}=\left.\int_{\Gamma} \boldsymbol{s}^{\mathrm{T}} \mathbf{L}^{\mathrm{T}} \overline{\mathbf{N}}\right|_{\Gamma} \mathrm{d} s=\left.\sum_{i=1}^{n} \int_{\Gamma_{i}} \mathbf{s}(x, y)^{\mathrm{T}} \mathbf{L}^{\mathrm{T}} \overline{\mathbf{N}}\right|_{\Gamma_{i}} \mathrm{~d} s .
$$

And the direction cosines of the outer normal of each element edge, $l$ and $m$ in (2), are given by

$$
l=\frac{\mathrm{d} y}{\mathrm{~d} s}, \quad m=-\frac{\mathrm{d} x}{\mathrm{~d} s} .
$$

Substitution of (15) and (16) into (18) yields

$$
\mathbf{H}=\left.\sum_{i=1}^{n} \int_{-1}^{1} \mathbf{S}(\xi)^{\mathrm{T}} \widetilde{\mathbf{L}}^{\mathrm{T}} \overline{\mathbf{N}}\right|_{\Gamma_{i}} \mathrm{~d} \xi
$$

with

$$
\begin{gathered}
\widetilde{\mathbf{L}}=\left[\begin{array}{ccc}
\frac{\mathrm{d} y}{\mathrm{~d} \xi} & 0 & -\frac{\mathrm{d} x}{\mathrm{~d} \xi} \\
0 & -\frac{\mathrm{d} x}{\mathrm{~d} \xi} & \frac{\mathrm{d} y}{\mathrm{~d} \xi}
\end{array}\right], \\
\mathrm{d} x=\mathrm{d} \bar{N}_{1}(\xi) x_{i}+\mathrm{d} \bar{N}_{2}(\xi) x_{j}+\mathrm{d} \bar{N}_{3}(\xi) x_{k}, \\
\mathrm{~d} y=\mathrm{d} \bar{N}_{1}(\xi) y_{i}+\mathrm{d} \bar{N}_{2}(\xi) y_{j}+\mathrm{d} \bar{N}_{3}(\xi) y_{k} .
\end{gathered}
$$

Five Gauss integration points are used to evaluate (18).
3.3. Evaluation Procedure for the Matrix $M$ in (8). As shown in Figure 2, any polygonal element can be divided into some subtriangles. The matrix $\mathbf{M}$ in (8) will be firstly evaluated in each subtriangle by standard numerical integration technique. And the sum of these values is the final matrix $\mathbf{M}$. The whole procedure can be performed automatically by computer code.

The resulting new polygonal element is denoted by HSFAP-19 $\beta$.

\section{Numerical Examples}

In this section, several problems are solved to test the performance of the new quadratic polygonal HSF-AP- $19 \beta$ element. The results are compared with those obtained by the following two elements:

PS2: the quadratic polygonal spline element based on area coordinates and B-net method, Chen et al. [32];

BFEM: the base force element method for quadratic polygonal mesh problems, Peng et al. [37, 38].

4.1. Patch Test. A small patch is divided into some arbitrary polygonal elements in Figure 3. The displacement fields and the exact stress solution corresponding to the constant strain are

$$
\begin{gathered}
u=10^{-3}\left(x+\frac{y}{2}\right), \quad v=10^{-3}\left(y+\frac{x}{2}\right), \\
\sigma_{x}=\sigma_{y}=1333.3333, \quad \tau_{x y}=400.0 .
\end{gathered}
$$




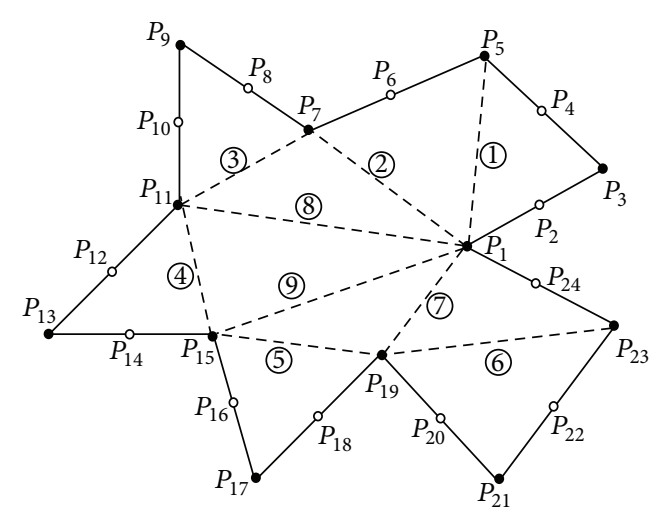

FIgURE 2: Domain divisions of a dodecagonal element.
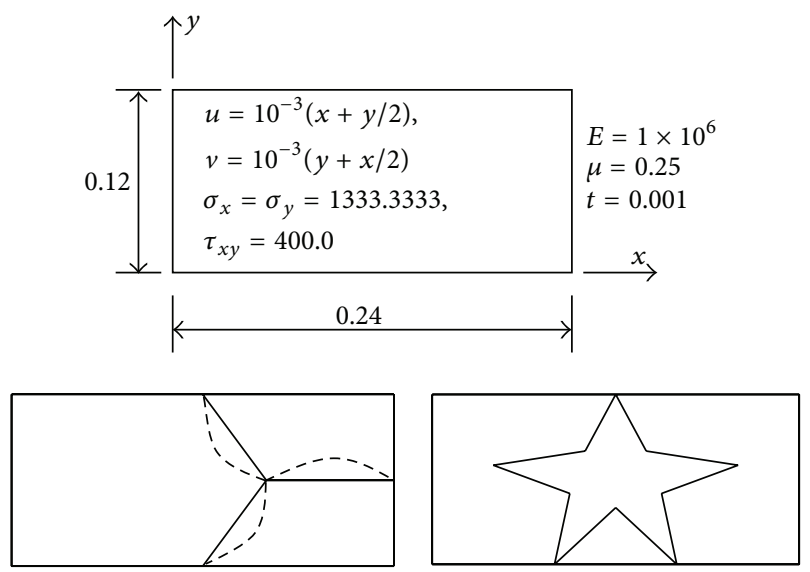

FIGURE 3: Constant stress/strain patch test.

The displacements of boundary nodes are taken as the displacement boundary conditions. No matter whether the inner element edges are straight or curved and no matter whether the shapes of the elements are convex or concave, the exact results of the displacements at each node and stresses at any point can be obtained by the present HSF-AP- $15 \beta$ element. This demonstrates that the new element passes the patch test and thus can ensure solution convergence.

4.2. Pure Shear Test of a Rectangular Plate. As shown in Figure 4, several polygonal meshes are applied to analyze a cantilever rectangular plate subjected to uniformly distributing shearing forces. Consider the plane stress condition; the parameters of the model are taken as $a=8, b=5, E=1$, $\mu=0.3$, and $\tau_{0}=1.0$. It can be seen that no matter whether the element shapes in the meshes are convex or concave polygonal, the following exact solutions [2],

$$
\tau_{x y}=1,\left.\quad v\right|_{x=a}=7.8,
$$

can be obtained.

4.3. Pure Bending Problem of a Cantilever Beam. As shown in Figure 5, a cantilever beam under plane stress condition is

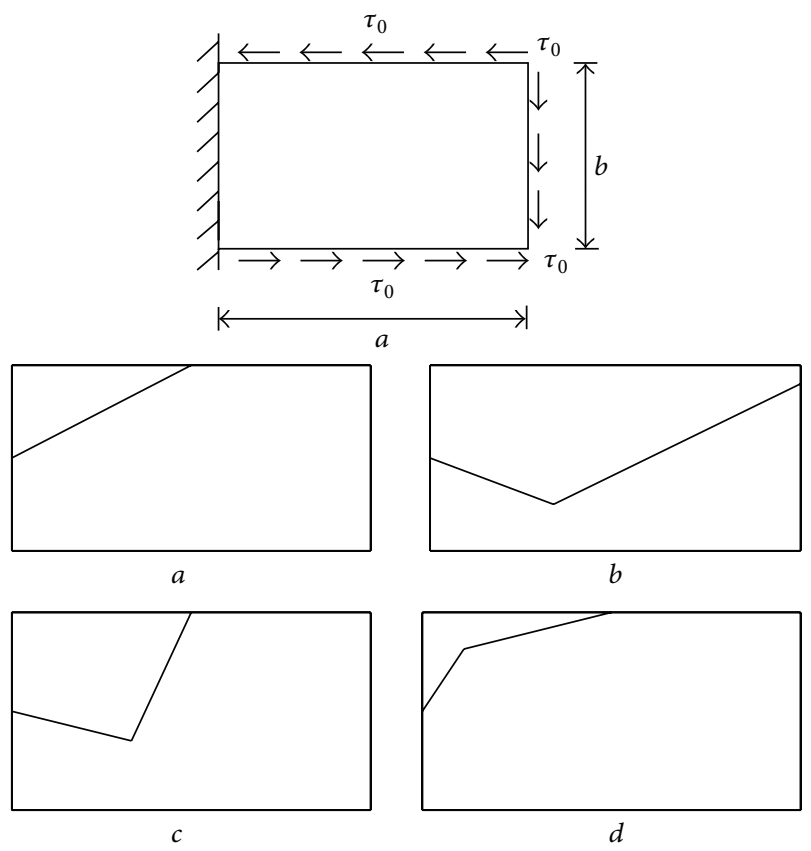

FIGURE 4: Rectangular plate subjected to shearing forces.

subjected to a constant bending moment $M$. The theoretical solutions for this problem are given by [48]

$$
\begin{gathered}
\sigma_{x}=\frac{12}{c} y-6, \quad \sigma_{y}=0, \quad \tau_{x y}=0, \\
u=-\frac{6}{E} x+\frac{12}{c E} x y, \quad v=\frac{6 \mu}{E} y-\frac{6}{c E} x^{2}-\frac{6 \mu}{c E} y^{2} .
\end{gathered}
$$

The dimensions of the beam are $L=10$ and $c=1$. Other parameters are given in Figure 5. Eighteen mesh divisions containing polygonal elements with concave and convex shapes are employed for the calculations. The displacement and stress results of select points are listed in Table 2.

It can be seen that, so long as all element edges keep straight, exact displacements and stress solutions can always be obtained by the element HSF-AP-19 $\beta$. However, even if a large number of meshes are employed to solve this problem, the exact solutions cannot be obtained by the BFEM [37].

4.4. Linear Bending for a Cantilever Beam. As shown in Figure 6, a cantilever under plane stress condition beam is subjected to a linear bending moment caused by a shear force $P$ at the free end. The theoretical solutions for this problem are given by [48]

$$
\begin{gathered}
\sigma_{x}=\frac{240}{c L} x y-\frac{120}{L} x-\frac{240}{c} y+120, \quad \sigma_{y}=0, \\
\tau_{x y}=-\frac{120}{c} y^{2}+\frac{120}{L} y, \\
u=\left(\frac{120}{c L} x^{2} y-\frac{92}{c L} y^{3}-\frac{60}{L} x^{2}-\frac{240}{c} x y\right. \\
\left.+\frac{138}{L} y^{2}+120 x-\frac{46 c}{L} y\right)(E)^{-1}
\end{gathered}
$$




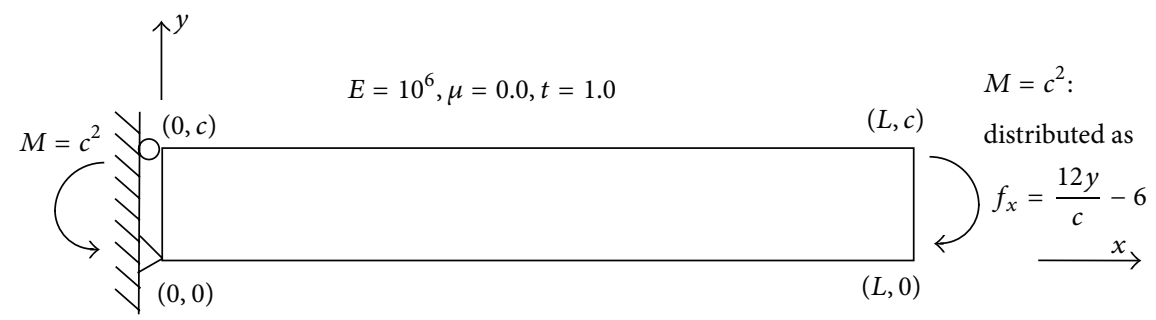

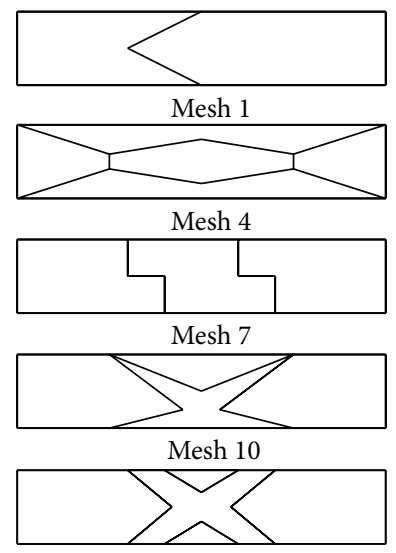

Mesh 13

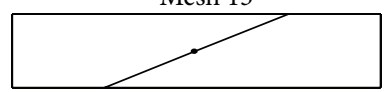

Mesh 16

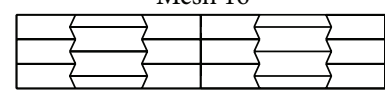

Mesh 19

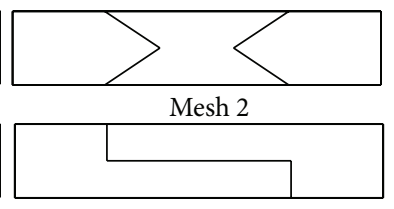

Mesh 5

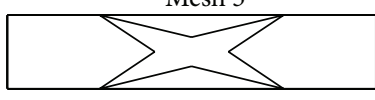

Mesh 8

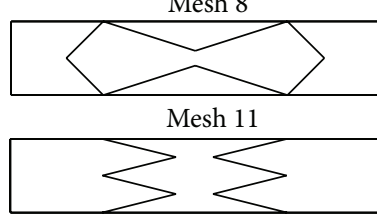

Mesh 14

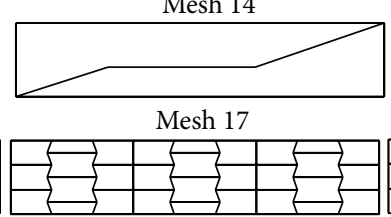

Mesh 20

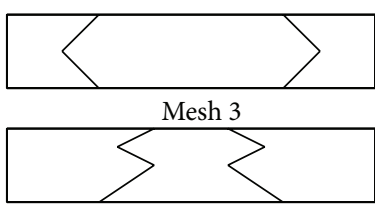

Mesh 6
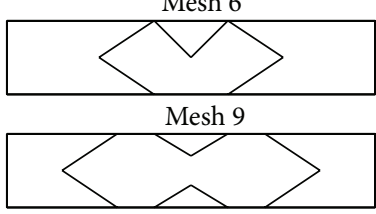

Mesh 12

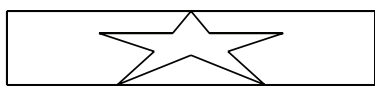

Mesh 15

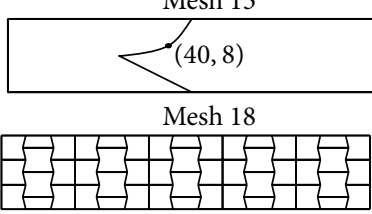

Mesh 21

Figure 5: Pure bending problem for a cantilever beam.

$$
\begin{gathered}
v=\left(-\frac{40}{c L} x^{3}-\frac{36}{c L} x y^{2}+\frac{120}{c} x^{2}+\frac{36}{L} x y\right. \\
\left.+\frac{36}{c} y^{2}+\frac{46 c}{L} x-36 y\right)(E)^{-1} .
\end{gathered}
$$

The mesh divisions employed are given in Figure 6. All numerical results are listed in Table 3. For this linear bending problem, the exact solutions cannot be directly obtained by the present HSF-AP-19 $\beta$ element. However, once the upper and lower boundaries of the beam are divided into more segments, the results will rapidly converge to the exact solutions.

4.5. MacNeal's Thin Cantilever Beam Problem [45]. As shown in Figure 7, six new mesh divisions are designed to solve thin beam problem proposed by MacNeal and Harder [45]. Two loading cases are considered: pure bending and transverse linear bending. Young's modulus $E=10^{7}$, Poisson's ratio $\mu=0.3$, and the thickness of the beam $t=0.1$. The results of the tip deflection are given in Table 4 . It can be seen that the present HSF-AP-19 $\beta$ element exhibits good performance: it can not only provide the exact solutions for the pure bending case but also produce the high precision results for the linear bending case.
4.6. Cook's Skew Beam Problem [49]. As shown in Figure 8, a skew cantilever under plane stress condition is subjected to a shear distributed load at the free edge. The typical meshes are given in Figure 8. The results of vertical deflection at point $C(48,52)$, the maximum principal stress at point $A(24,22)$, and the minimum principal stress at point $B(24,52)$ are all listed in Table 5 , in which the stresses are the average values at the corresponding nodes. It can be seen that the new HSFAP-19 $\beta$ element has a good convergence.

4.7. Curved Beam. As shown in Figure 9, a curved cantilever beam under plane stress condition is subjected to a transverse force $P$ at the upper end in the radial direction. The dimensions of the model are as follows: the inner radius $a=0.7$, the outer radius $b=1.0$, and other constants are given in Figure 9. Two Poisson's ratio cases are considered: $\mu=0.3$ and $\mu=0.4999$. Furthermore, two mesh division types are adopted: elements with curved edges and straight edges. The theoretical radial displacement of point $A$ at the upper end is given by [48]

$$
v_{A}=-\frac{P \pi\left(a^{2}+b^{2}\right)}{E\left[\left(a^{2}-b^{2}\right)+\left(a^{2}+b^{2}\right) \log (b / a)\right]} .
$$

The results of the tip displacement $v_{A}$ are given in Table 6 . It can be observed that the new HSF-AP-19 $\beta$ element exhibits 


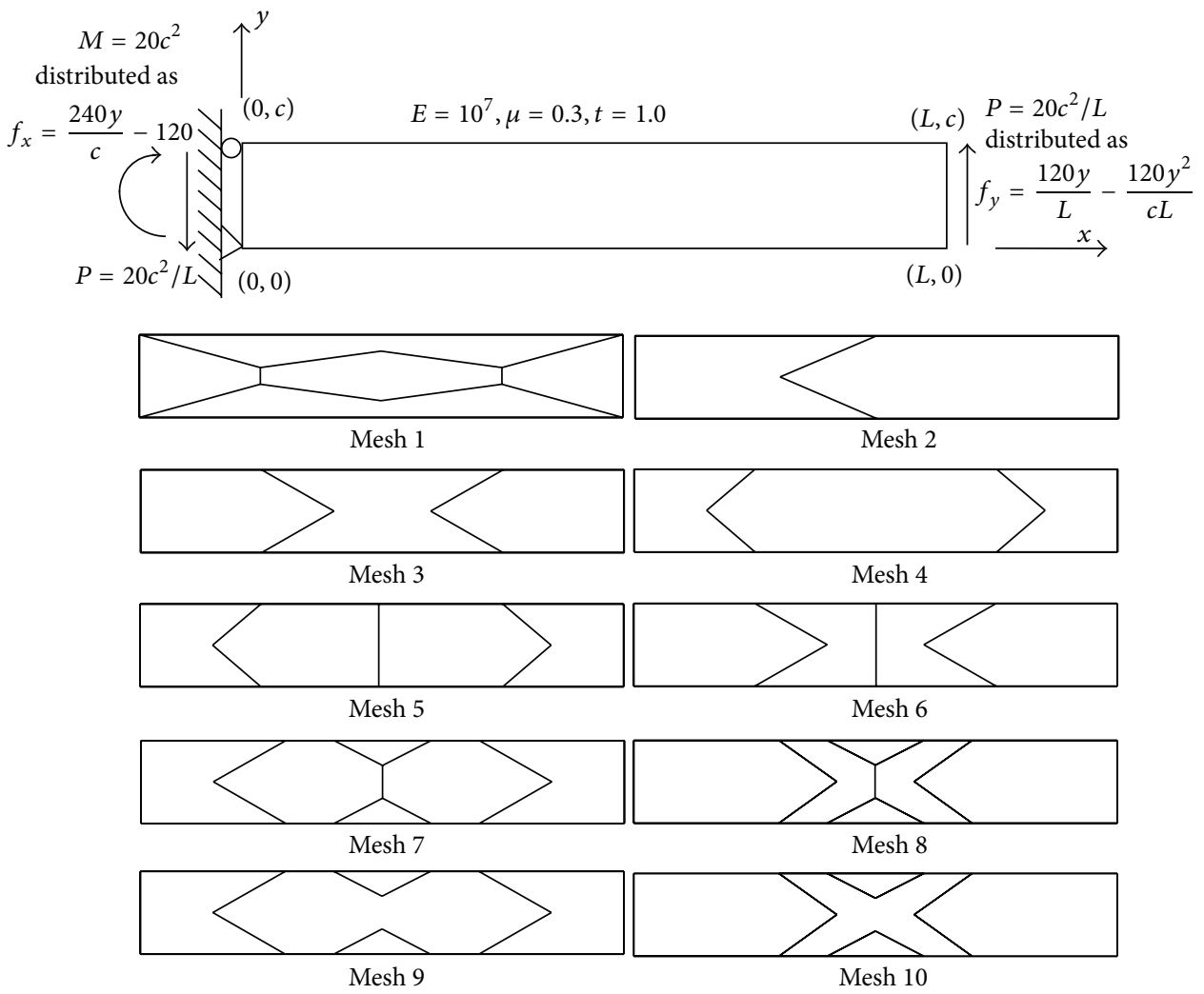

FIGURE 6: Linear bending problem for a cantilever beam.

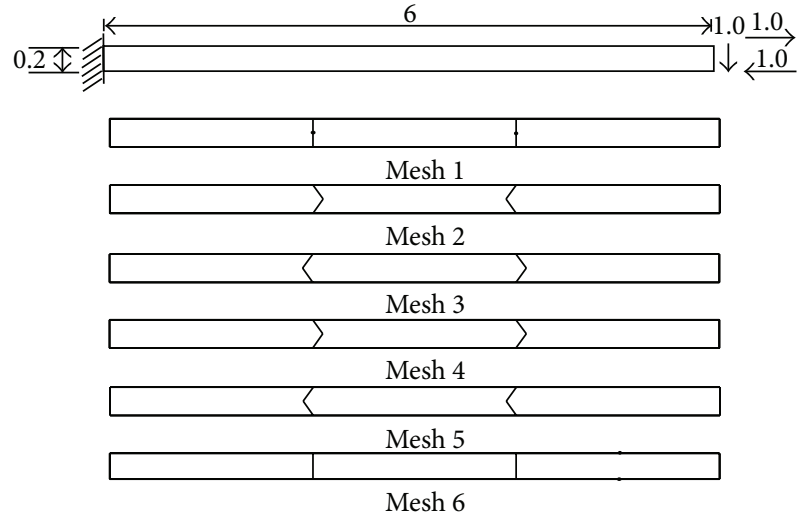

Figure 7: MacNeal's thin beam problem.

good convergence. Moreover, the errors produced by the HSF-AP-19 $\beta$ element are quit small, no matter whether the meshes have curved or straight edges.

4.8. Infinite Plate with a Circular Hole [3]. As shown in Figure 10, a plate with a central circular hole is under plane stress state and is subjected to a uniform tensile load of 1.0 in $x$-direction. In order to simulate the properties of the infinite plate, the plate-to-hole aspect ratio of 50 is applied, and the radius of hole is taken as 1.0 , while the half-width of plate is 50. The related parameters are as follows: $E=1.0 \times 10^{3}$, $\mu=0.3$, and thickness $t=1.0$. The meshes employed are given in Figure 10.

The results at points $A$ and $B$ are listed in Table 7 . Besides the HSF-AP-19 $\beta$ element, the results obtained by the PS2 [32] element are also given for comparison. It can be seen that the accuracy of the results obtained by the HSF-AP-19 $\beta$ element is higher than those obtained by the PS2 element, especially for stress solutions.

4.9. A Cantilever Beam under a Concentrated Load. As shown in Figure 11, a cantilever beam with large aspect ratio is subjected to a concentrated load $P=1$ at free end. The geometric parameters of the cantilever beam are as follows: $L=5, t=1$, and $h=0.1$. Young's modulus is $E=10^{9}$, and Poisson's ratio is zero. Six mesh divisions are shown in Figure 11, in which Meshes a, b, and c are employed for the HSF-AP-19 $\beta$ element and Meshes $d, e$, and $f$ for the BFEM [37].

The vertical displacements of point $A$ are presented in Table 8. It can be seen that the HSF-AP-19 $\beta$ element can provide better results by using much less elements.

4.10. Cook's Skew Beam Problem with Heterogeneous Material. As shown in Figure 12, a Cook's skew beam with heterogeneous material (under plane stress condition) is subjected to a shear distributed load $P$ at the free edge. The beam is 

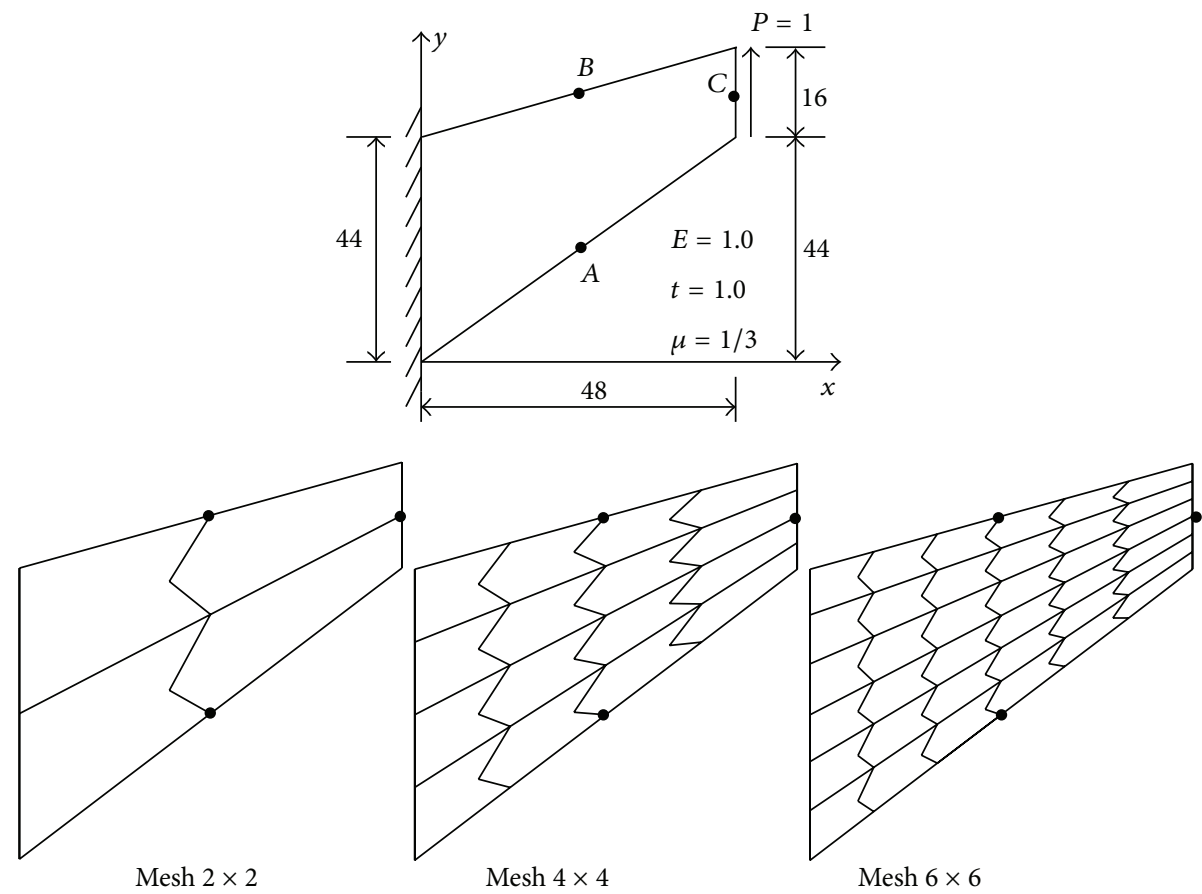

FIGURE 8: Cook’s skew beam problem.

TABLE 2: Results at selected locations for the pure bending problem (Figure 5).

\begin{tabular}{lcccc}
\hline Models & Meshes & $\sigma_{x}(0,0)$ & $\sigma_{x}(0,1)$ & $v(10,0) \times 10^{-4}$ \\
\hline & Mesh 1 & -6.000 & 6.000 & -6.0000 \\
& Mesh 2 & -6.000 & 6.000 & -6.0000 \\
& Mesh 3 & -6.000 & 6.000 & -6.0000 \\
& Mesh 4 & -6.000 & 6.000 & -6.0000 \\
& Mesh 5 & -6.000 & 6.000 & -6.0000 \\
& Mesh 6 & -6.000 & 6.000 & -6.0000 \\
HSF-AP-19 $\beta$ & Mesh 7 & -6.000 & 6.000 & -6.0000 \\
& Mesh 8 & -6.000 & 6.000 & -6.0000 \\
& Mesh 9 & -6.000 & 6.000 & -6.0000 \\
& Mesh 10 & -6.000 & 6.000 & -6.0000 \\
& Mesh 12 & -6.000 & 6.000 & -6.0000 \\
& Mesh 13 & -6.000 & 6.000 & -6.0000 \\
& Mesh 14 & -6.000 & 6.000 & -6.0000 \\
& Mesh 15 & -6.000 & 6.000 & -6.0000 \\
& Mesh 16 & -6.000 & 6.000 & -6.0000 \\
BFEM [37] & Mesh 17 & -6.000 & 6.000 & -6.00000 \\
& Mesh 18 & -6.048 & 6.089 & -5.9972 \\
\hline & Mesh 19 & - & - & -6.0339 \\
& Mesh 21 & - & - & -6.0359 \\
& & -6.000 & 6.000 & -6.0000 \\
\hline & & & &
\end{tabular}

heterogeneous and made of two materials: (i) $E=1.0, \mu=$ 0.25 and (ii) $E=1.5, \mu=0.3$. Two heterogeneous models are

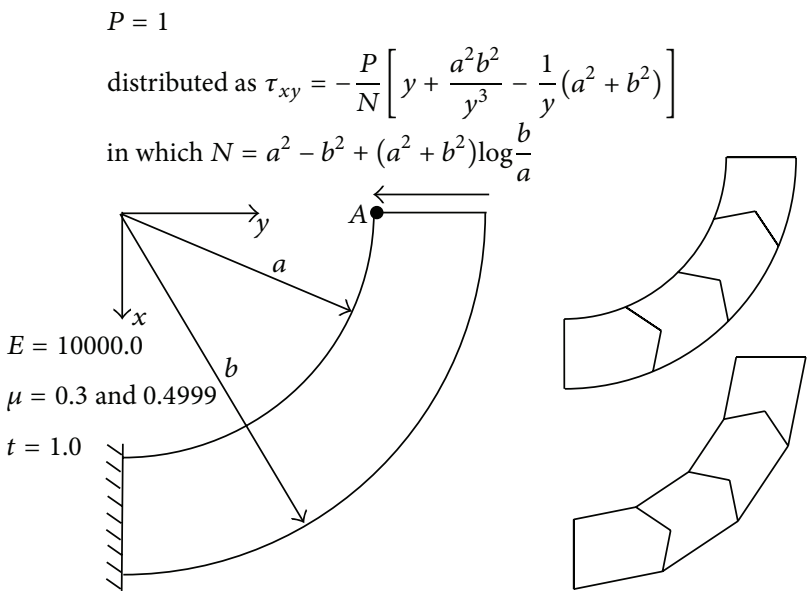

FIGURE 9: Curved beam problem.

considered here, and the corresponding mesh divisions are given in Figures 13(a) and 13(b), in which the gray domains are made of the first material, while other domains are made of the second material. The results of vertical deflection at point $C(48,52)$ are listed in Table 9.

With the mesh refinement, the results obtained by Abaqus 8-node elements CPS8 and CPS8R [47] will tend to the exact solutions. As to a specified precision, the total element number needed by Abaqus is much more than that required by the HSF-AP- $19 \beta$ element. This example is a very simple problem of heterogeneous materials. However, when solids with more complicated materials are modeled, such as the crystal structures, the computational 
TABLE 3: Results obtained by the element HSF-AP-19 $\beta$ at selected locations for the linear bending problem (Figure 6).

\begin{tabular}{|c|c|c|c|c|c|c|}
\hline & \multicolumn{2}{|c|}{$v(100,0) \times 10^{-3}$} & \multicolumn{2}{|c|}{$\sigma(0,0)$} & \multicolumn{2}{|c|}{$\sigma(0,10)$} \\
\hline & Numerical results & Normalized results & Numerical results & Normalized results & Numerical results & Normalized results \\
\hline Mesh 1 & 6.340 & 0.788 & 67.57 & 0.563 & -67.34 & 0.561 \\
\hline Mesh 2 & 7.834 & 0.974 & 102.16 & 0.851 & -101.94 & 0.850 \\
\hline Mesh 3 & 7.915 & 0.984 & 112.57 & 0.938 & -113.12 & 0.943 \\
\hline Mesh 4 & 7.911 & 0.983 & 112.88 & 0.941 & -112.56 & 0.938 \\
\hline Mesh 5 & 8.026 & 0.998 & 114.18 & 0.952 & -113.66 & 0.947 \\
\hline Mesh 6 & 8.035 & 0.999 & 115.74 & 0.965 & -116.11 & 0.968 \\
\hline Mesh 7 & 8.033 & 0.998 & 113.44 & 0.945 & -113.10 & 0.943 \\
\hline Mesh 8 & 8.062 & 1.002 & 117.59 & 0.980 & -117.53 & 0.979 \\
\hline Mesh 9 & 8.055 & 1.001 & 115.67 & 0.964 & -118.93 & 0.991 \\
\hline Mesh 10 & 8.066 & 1.002 & 117.82 & 0.982 & -117.84 & 0.982 \\
\hline Exact & \multicolumn{2}{|c|}{8.046} & \multicolumn{2}{|c|}{120.00} & \multicolumn{2}{|c|}{-120.00} \\
\hline
\end{tabular}

TABLE 4: Results at selected locations for MacNeal's thin beam problem (Figure 7).

\begin{tabular}{lcccc}
\hline & & Load $P$ & \multicolumn{2}{c}{ Load $M\left(\times 10^{-02}\right)$} \\
& Numerical results & Normalized results & Numerical results & Normalized results \\
\hline Mesh 1 & -0.1060 & 0.9806 & -0.5400 & 1.0000 \\
Mesh 2 & -0.1060 & 0.9806 & -0.5400 & 1.0000 \\
Mesh 3 & -0.1060 & 0.9806 & -0.5400 & 1.0000 \\
Mesh 4 & -0.1060 & 0.9806 & -0.5400 & 1.0000 \\
Mesh 5 & -0.1060 & 0.9806 & -0.5400 & 1.0000 \\
Mesh 6 & -0.1064 & 0.9843 & -0.5400 & 1.0000 \\
\hline [45] & & & -0.5400
\end{tabular}

TABLE 5: Results at selected locations for Cook's skew beam problem (Figure 8).

\begin{tabular}{lccc}
\hline Mesh & $v_{C}$ & $\sigma_{A \max }$ & $\sigma_{B \min }$ \\
\hline $2 \times 2$ & 23.79 & 0.2473 & -0.1761 \\
$4 \times 4$ & 23.94 & 0.2406 & -0.1946 \\
$6 \times 6$ & 23.95 & 0.2386 & -0.2018 \\
$8 \times 8$ & 23.96 & 0.2378 & -0.2033 \\
\hline Reference solution $^{\mathrm{a}}$ & 23.96 & 0.2362 & -0.2023 \\
\hline
\end{tabular}

${ }^{\text {a } R e s u l t s ~ o f ~ t h e ~ e l e m e n t ~ G T 9 M 8 ~[46] ~ u s i n g ~} 64 \times 64$ mesh.

TABLE 6: The tip displacements $v_{A}$ of the curved beam (Figure 9).

\begin{tabular}{lcccc}
\hline & \multicolumn{2}{c}{ Curved edges } & \multicolumn{2}{c}{ Straight edges } \\
& $\mu=0.3$ & $\mu=0.4999$ & $\mu=0.3$ & $\mu=0.4999$ \\
\hline $1 \times 2$ & -0.0211 & -0.0211 & -0.0223 & -0.0222 \\
$1 \times 4$ & -0.0217 & -0.0216 & -0.0218 & -0.0217 \\
$1 \times 6$ & -0.0217 & -0.0216 & -0.0217 & -0.0216 \\
$1 \times 8$ & -0.0217 & -0.0216 & -0.0217 & -0.0216 \\
$1 \times 10$ & -0.0217 & -0.0217 & -0.0217 & -0.0217 \\
\hline Analytical solution & \multicolumn{3}{c}{-0.0218} \\
\hline
\end{tabular}

cost of the HSF-AP-19 $\beta$ element will be much less than that of CPS8 and CPS8R in Abaqus. Therefore, it can be said that the new polygonal element exhibits obvious
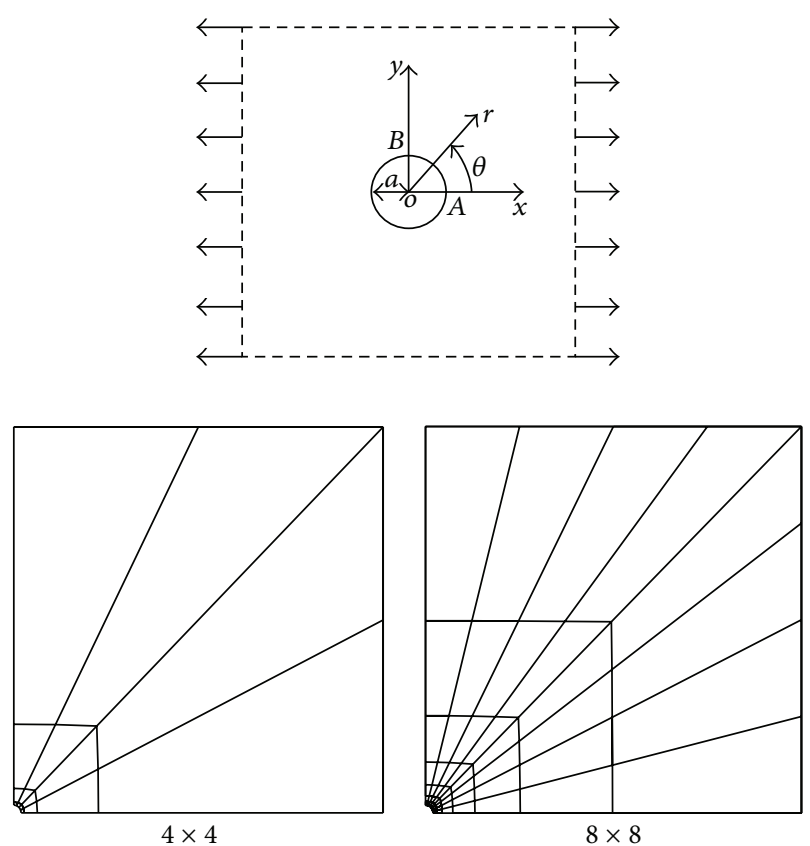

FIGURE 10: Infinite plate with a circular hole.

advantages in the analysis of complex heterogeneous material problems. 
TABle 7: The results at points $A$ and $B$ of the infinite plate with a circular hole (Figure 10).

\begin{tabular}{|c|c|c|c|c|c|c|}
\hline & Mesh & $u(A) / 10^{-3}$ & $v(B) / 10^{-3}$ & $\sigma_{y}(A)$ & $\sigma_{x}(B)$ & Number of nodes \\
\hline \multirow{5}{*}{ HSF-AP- $19 \beta$} & $4 \times 4$ & 2.992 & -0.999 & -1.058 & 3.045 & 65 \\
\hline & $8 \times 8$ & 3.002 & -1.002 & -1.007 & 3.008 & 225 \\
\hline & $12 \times 12$ & 3.003 & -1.003 & -1.004 & 3.006 & 481 \\
\hline & $16 \times 16$ & 3.003 & -1.002 & -1.003 & 3.003 & 883 \\
\hline & $20 \times 20$ & 3.003 & -1.002 & -1.002 & 3.002 & 1281 \\
\hline \multirow{3}{*}{ PS2 [32] } & $4 \times 4$ & 2.917 & -0.950 & -1.025 & 3.095 & 65 \\
\hline & $8 \times 8$ & 2.985 & -0.993 & -1.026 & 3.044 & 225 \\
\hline & $12 \times 12$ & 2.994 & -0.998 & -1.013 & 3.021 & 481 \\
\hline Exact & & 3.000 & -1.000 & -1.000 & 3.000 & \\
\hline
\end{tabular}

TABLE 8: The vertical displacements at point $A$ of the cantilever beam under a concentrated load (Figure 11).

\begin{tabular}{|c|c|c|c|c|c|}
\hline \multirow{2}{*}{ Mesh } & \multicolumn{2}{|c|}{ HSF-AP-19 $\beta$} & \multicolumn{2}{|c|}{ BFEM } & \multirow{2}{*}{ Analytical solution } \\
\hline & Numerical results & Relative errors & Numerical results & Relative errors & \\
\hline $\mathrm{a}$ & -4.7915 & $4.1696 \%$ & & \multirow{6}{*}{-5.0000} \\
\hline $\mathrm{b}$ & -4.9561 & $0.8772 \%$ & & & \\
\hline c & \multirow{4}{*}{\multicolumn{2}{|c|}{$0.4230 \%$}} & & & \\
\hline d & & & -4.9787 & $0.4260 \%$ & \\
\hline $\mathrm{e}$ & & & -5.0159 & $0.3180 \%$ & \\
\hline $\mathrm{f}$ & & & -4.9734 & $0.5320 \%$ & \\
\hline
\end{tabular}

TABLE 9: The vertical displacements at point $C$ of Cook's skew beam problem with heterogeneous materials (Figures 12 and 13 ).

\begin{tabular}{|c|c|c|c|c|c|}
\hline Heterogeneous model & Mesh & Number of elements/nodes & HSF-AP-19 $\beta$ & CPS8 [47] & CPS8R [47] \\
\hline \multirow{6}{*}{ The first case (a) } & Mesh 1 & $9 / 48$ & 18.7076 & - & - \\
\hline & Mesh 2 & $15 / 64$ & \multirow{5}{*}{-} & 18.6115 & 18.5847 \\
\hline & Mesh 3 & $50 / 177$ & & 18.6890 & 18.7002 \\
\hline & Mesh 4 & $77 / 264$ & & 18.6957 & 18.7000 \\
\hline & Mesh 5 & $112 / 383$ & & 18.7227 & 18.7200 \\
\hline & Mesh 6 & $424 / 1365$ & & 18.7408 & 18.7422 \\
\hline \multirow{5}{*}{ The second case (b) } & Mesh 1 & $27 / 128$ & \multirow[t]{5}{*}{19.4072} & - & - \\
\hline & Mesh 2 & $112 / 382$ & & 19.3741 & 19.3846 \\
\hline & Mesh 3 & $245 / 802$ & & 19.3992 & 19.4037 \\
\hline & Mesh 4 & $424 / 1365$ & & 19.4062 & 19.4080 \\
\hline & Mesh 5 & $764 / 2413$ & & 19.4103 & 19.4114 \\
\hline
\end{tabular}

\section{Concluding Remarks}

In the paper, a novel quadratic hybrid stress-function polygonal element model, HSF-AP-19 $\beta$, is developed based on the principle of minimum complementary energy and the fundamental analytical solutions of the Airy stress function. The present element has the following characters.

(1) It is simple to build this new element without construction of displacement interpolation functions, which are very complex for polygonal elements compared with quadrilateral elements. This polygonal HSF-AP-19 $\beta$ element can be degenerated into triangular or quadrilateral elements directly.

(2) Spurious zero energy modes can be avoided by selecting suitable number of fundamental analytical solutions for stresses (from constant terms to higherorder terms). For quadratic HS-F model, the first nineteen stress solutions must be employed.

(3) The present HSF-AP-19 $\beta$ element exhibits better accuracy and convergence in both displacement and stress solutions. Even though meshes containing convex or concave elements are employed, the precisions of the results will not be affected. So, it is a shape-free element model. For constant stress/strain problem, the exact solutions can be obtained for all cases. So long as element edges keep straight, all elements can produce the exact solutions for pure bending problem. Moreover, the new element can produce better results using relatively coarse meshes for some problems, such as high-order problems, problems 

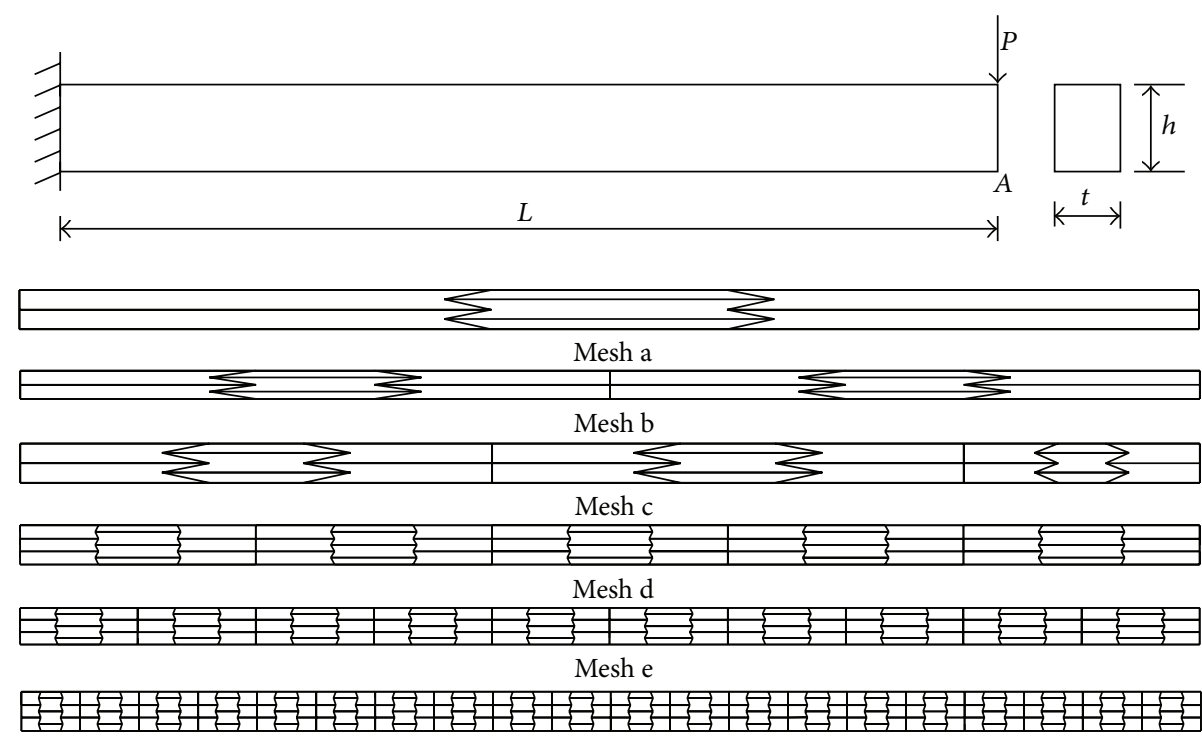

Mesh $\mathrm{f}$

FIGURE 11: A cantilever beam under a concentrated load.

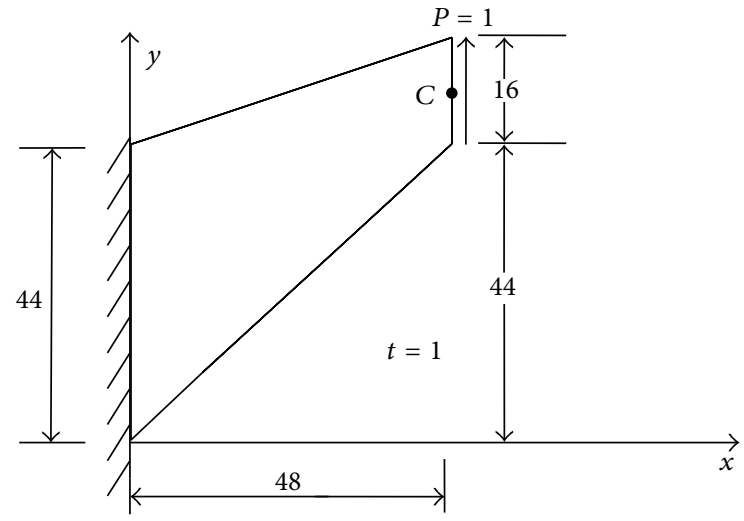

FIGURE 12: Cook's skew beam problem with heterogeneous material.

with circular holes, and heterogeneous material problems.

\section{Conflict of Interests}

The authors declare that there is no conflict of interests regarding the publication of this paper.

\section{Acknowledgments}

The authors would like to acknowledge the financial support of the National Natural Science Foundation of China (11272181), the Specialized Research Fund for the Doctoral Program of Higher Education of China (20120002110080), and the National Basic Research Program of China (Project no. 2010CB832701).

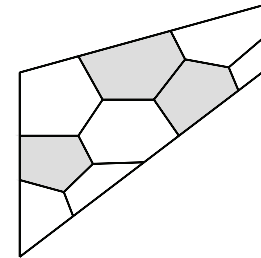

Mesh 1

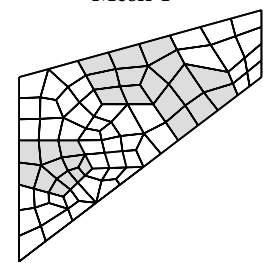

Mesh 4

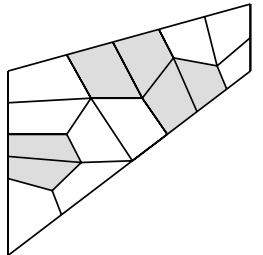

Mesh 2

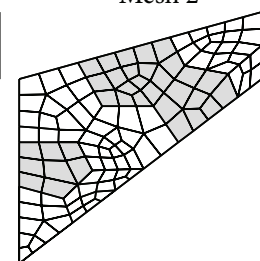

Mesh 5

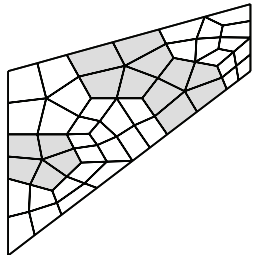

Mesh 3

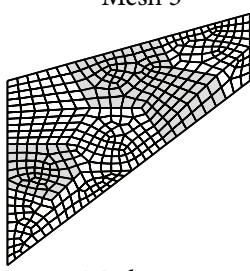

Mesh 6 (a) Meshes for the first heterogeneous model

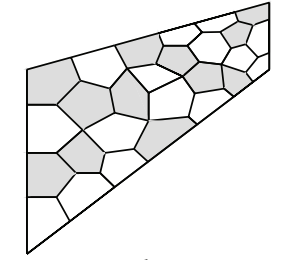

Mesh 1

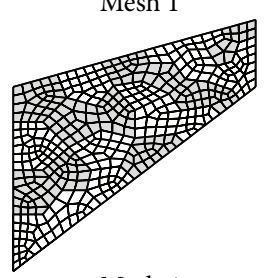

Mesh 4

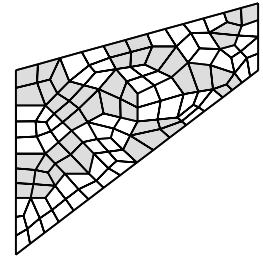

Mesh 2

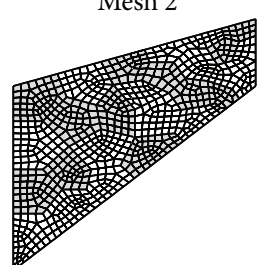

Mesh 5

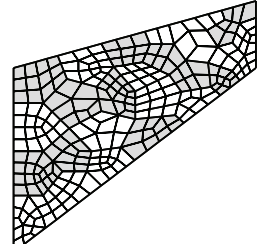

Mesh 3 (b) Meshes for the second heterogeneous model

FIGURE 13: Meshes divisions for the heterogeneous models. 


\section{References}

[1] Y. Q. Long, S. Cen, and Z. F. Long, Advanced Finite Element Method in Structural Engineering, Springer, Berlin, Germany; Tsinghua University Press, Beijing, China, 2009.

[2] O. C. Zienkiewicz and R. L. Taylor, The Finite Element Method for Solid and Structural Mechanics, Elsevier ButterworthHeinnemann, Oxford, UK, 6th edition, 2005.

[3] Y. L. Fan, Y. G. Zhang, and M. W. Lu, "Arbitrarily polygonal 2-D finite element," Acta Mechanica Sinica, vol. 27, no. 6, pp. 742746, 1995 (Chinese).

[4] R. B. Martin, D. B. Burr, and N. A. Sharkey, Skeletal Tissue Mechanics, Springer, New York, NY, USA, 1998.

[5] E. Cueto, B. Calvo, and M. Doblaré, "Modelling threedimensional piece-wise homogeneous domains using the $\alpha$ shape-based natural element method," International Journal for Numerical Methods in Engineering, vol. 54, no. 6, pp. 871-897, 2002.

[6] A. R. Diaz and A. Bénard, "Designing materials with prescribed elastic properties using polygonal cells," International Journal for Numerical Methods in Engineering, vol. 57, no. 3, pp. 301-314, 2003.

[7] C. R. Dohrmann, S. W. Key, and M. W. Heinstein, "A method for connecting dissimilar finite element meshes in two dimensions," International Journal for Numerical Methods in Engineering, vol. 48, no. 5, pp. 655-678, 2000.

[8] J. F. Peters and E. Heymsfield, "Application of the 2-D constant strain assumption to FEM elements consisting of an arbitrary number of nodes," International Journal of Solids and Structures, vol. 40, no. 1, pp. 143-159, 2003.

[9] S. Ghosh and S. Moorthy, "Elastic-plastic analysis of arbitrary heterogeneous materials with the Voronoi Cell finite element method," Computer Methods in Applied Mechanics and Engineering, vol. 121, no. 1-4, pp. 373-409, 1995.

[10] S. Ghosh, K. Lee, and S. Moorthy, "Multiple scale analysis of heterogeneous elastic structures using homogenization theory and voronoi cell finite element method," International Journal of Solids and Structures, vol. 32, no. 1, pp. 27-62, 1995.

[11] K. Lee and S. Ghosh, "Small deformation multi-scale analysis of heterogeneous materials with the Voronoi cell finite element model and homogenization theory," Computational Materials Science, vol. 7, no. 1-2, pp. 131-146, 1996.

[12] S. Moorthy and S. Ghosh, "Adaptivity and convergence in the Voronoi cell finite element model for analyzing heterogeneous materials," Computer Methods in Applied Mechanics and Engineering, vol. 185, no. 1, pp. 37-74, 2000.

[13] H. W. Zhang, H. Wang, and J. B. Wang, "Parametric variational principle based elastic-plastic analysis of materials with polygonal and Voronoi cell finite element methods," Finite Elements in Analysis and Design, vol. 43, no. 3, pp. 206-217, 2007.

[14] E. L. Wachspress, A Rational Finite Element Basis, Academic Press, New York, NY, USA, 1975.

[15] P. L. Powar and R. Rao, "A counterexample of the construction of $\mathrm{C}^{1}$-rational finite element due to Wachspress," Computers \& Mathematics with Applications, vol. 22, no. 3, pp. 17-22, 1991.

[16] H. P. Dikshit and A. Ojha, "Dimensions of spaces of Wachspress type $C^{1}$-rational finite elements," Computers and Mathematics with Applications, vol. 22, no. 3, pp. 23-26, 1991.

[17] M. R. Laydi and B. Aoubiza, "Wachspress rational finiteelements of arbitrary degree," Comptes Rendus de L Academie Des Sciences Serie I-Mathematique, vol. 320, no. 11, pp. 13911394, 1995.
[18] W. Dahmen, H. P. Dikshit, and A. Ojha, "On Wachspress quadrilateral patches," Computer Aided Geometric Design, vol. 17, no. 9, pp. 879-890, 2000.

[19] H. P. Dikshit and A. Ojha, "On $C^{1}$-continuity of Wachspress quadrilateral patches," Computer Aided Geometric Design, vol. 19, no. 3, pp. 207-222, 2002.

[20] H. P. Dikshit and A. Ojha, "A simple subdivision formula for quadrilateral Wachspress patches," Computer Aided Geometric Design, vol. 20, no. 7, pp. 395-399, 2003.

[21] G. Dasgupta, "Interpolants within convex polygons: wachspress' shape functions," Journal of Aerospace Engineering, vol. 16, no. 1, pp. 1-8, 2003.

[22] G. Dasgupta, "Integration within polygonal finite elements," Journal of Aerospace Engineering, vol. 16, no. 1, pp. 9-18, 2003.

[23] E. A. Malsch and G. Dasgupta, "Interpolations for temperature distributions: a method for all non-concave polygons," International Journal of Solids and Structures, vol. 41, no. 8, pp. 21652188, 2004.

[24] Z. Q. Wang and S. P. Li, "Some remarks on shape functions of polygonal finite element," Chinese Journal of Applied Mechanics, vol. 24, no. 4, pp. 604-608, 2007 (Chinese).

[25] N. Sukumar and A. Tabarraei, "Conforming polygonal finite elements," International Journal for Numerical Methods in Engineering, vol. 61, no. 12, pp. 2045-2066, 2004.

[26] C. T. Loop and T. D. Derose, "A multisided generalization of Bézier surfaces," ACM Transactions on Graphics, vol. 8, no. 3, pp. 204-234, 1989.

[27] M. S. Floater, "Mean value coordinates," Computer Aided Geometric Design, vol. 20, no. 1, pp. 19-27, 2003.

[28] M. S. Floater, K. Hormann, and G. Kós, "A general construction of barycentric coordinates over convex polygons," Advances in Computational Mathematics, vol. 24, no. 1-4, pp. 311-331, 2006.

[29] J. F. Peters and E. Heymsfield, "Application of the 2-D constant strain assumption to FEM elements consisting of an arbitrary number of nodes," International Journal of Solids and Structures, vol. 40, no. 1, pp. 143-159, 2003.

[30] G. R. Liu, T. Nguyen-Thoi, H. Nguyen-Xuan, and K. Y. Lam, "A node-based smoothed finite element method (NS-FEM) for upper bound solutions to solid mechanics problems," Computers and Structures, vol. 87, no. 1-2, pp. 14-26, 2009.

[31] K. Y. Dai, G. R. Liu, and T. T. Nguyen, "An $n$-sided polygonal smoothed finite element method (nSFEM) for solid mechanics," Finite Elements in Analysis and Design, vol. 43, no. 11-12, pp. 847860, 2007.

[32] J. Chen, C.-J. Li, and W.-J. Chen, "Construction of $n$-sided polygonal spline element using area coordinates and B-net method," Acta Mechanica Sinica, vol. 26, no. 5, pp. 685-693, 2010.

[33] N. Sukumar, "Quadratic maximum-entropy serendipity shape functions for arbitrary planar polygons," Computer Methods in Applied Mechanics and Engineering, vol. 263, pp. 27-41, 2013.

[34] S. Ghosh and R. L. Mallett, "Voronoi cell finite elements," Computers and Structures, vol. 50, no. 1, pp. 33-46, 1994.

[35] J. Zhang and N. Katsube, "A hybrid finite element method for heterogeneous materials with randomly dispersed elastic inclusions," Finite Elements in Analysis and Design, vol. 19, no. 1-2, pp. 45-55, 1995.

[36] J. Zhang and N. Katsube, "A hybrid finite element method for heterogeneous materials with randomly dispersed rigid inclusions," International Journal for Numerical Methods in Engineering, vol. 38, no. 10, pp. 1635-1653, 1995. 
[37] Y. J. Peng, L. J. Zhang, J. W. Pu, and Q. W. Guo, "A twodimensional base force element method using concave polygonal mesh," Engineering Analysis with Boundary Elements, vol. 42, pp. 45-50, 2014.

[38] Y. J. Peng, N. N. Zong, L. J. Zhang, and J. W. Pu, "Application of 2D base force element method with complementary energy principle for arbitrary meshes," Engineering Computations, vol. 31, no. 4, pp. 691-708, 2014.

[39] X.-R. Fu, S. Cen, C. F. Li, and X.-M. Chen, "Analytical trial function method for development of new 8-node plane element based on the variational principle containing Airy stress function," Engineering Computations, vol. 27, no. 4, pp. 442-463, 2010.

[40] S. Cen, X.-R. Fu, G.-H. Zhou, M.-J. Zhou, and C. F. Li, "Shapefree finite element method: the plane hybrid stress-function (HS-F) element method for anisotropic materials," Science China: Physics, Mechanics \& Astronomy, vol. 54, no. 4, pp. 653665, 2011.

[41] S. Cen, X.-R. Fu, and M.-J. Zhou, "8- and 12-node plane hybrid stress-function elements immune to severely distorted mesh containing elements with concave shapes," Computer Methods in Applied Mechanics and Engineering, vol. 200, no. 29-32, pp. 2321-2336, 2011.

[42] S. Cen, M.-J. Zhou, and X.-R. Fu, "A 4-node hybrid stressfunction (HS-F) plane element with drilling degrees of freedom less sensitive to severe mesh distortions," Computers and Structures, vol. 89, no. 5-6, pp. 517-528, 2011.

[43] M.-J. Zhou, S. Cen, Y. Bao, and C.-F. Li, "A quasi-static crack propagation simulation based on shape-free hybrid stressfunction finite elements with simple remeshing," Computer Methods in Applied Mechanics and Engineering, vol. 275, pp. 159-188, 2014.

[44] M.-J. Zhou, Shape-free plane hybrid stress-function finite element method [Ph.D. thesis], Tsinghua University, Beijing, China, 2014.

[45] R. H. MacNeal and R. L. Harder, "A proposed standard set of problems to test finite element accuracy," Finite Elements in Analysis and Design, vol. 1, no. 1, pp. 3-20, 1985.

[46] L. Yuqiu and X. Yin, "Generalized conforming triangular membrane element with vertex rigid rotational freedoms," Finite Elements in Analysis and Design, vol. 17, no. 4, pp. 259-271, 1994.

[47] Abaqus 6.9, HTML Documentation, Dassault Systèmes Simulia Corp., Providence, RI, USA, 2009.

[48] S. P. Timoshenko and J. N. Goodier, Theory of Elasticity, McGraw-Hill, New York, NY, USA, 3rd edition, 1934.

[49] R. D. Cook, D. S. Malkus, and M. E. Plesha, Concepts and Applications of Finite Element Analysis, John Wiley \& Sons, New York, NY, USA, 3rd edition, 1989. 


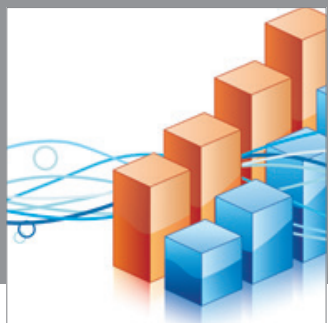

Advances in

Operations Research

mansans

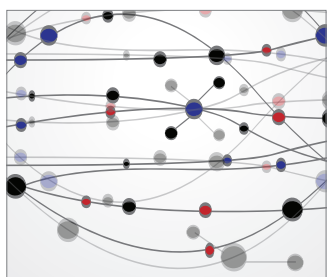

The Scientific World Journal
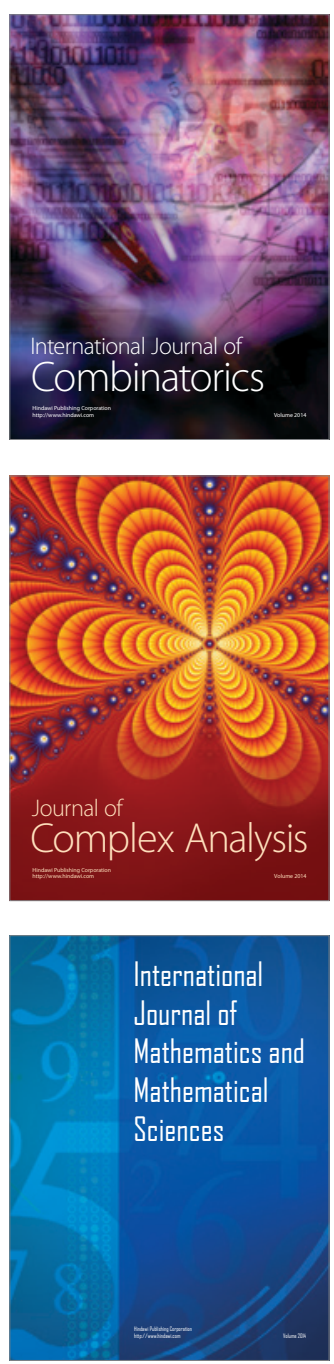
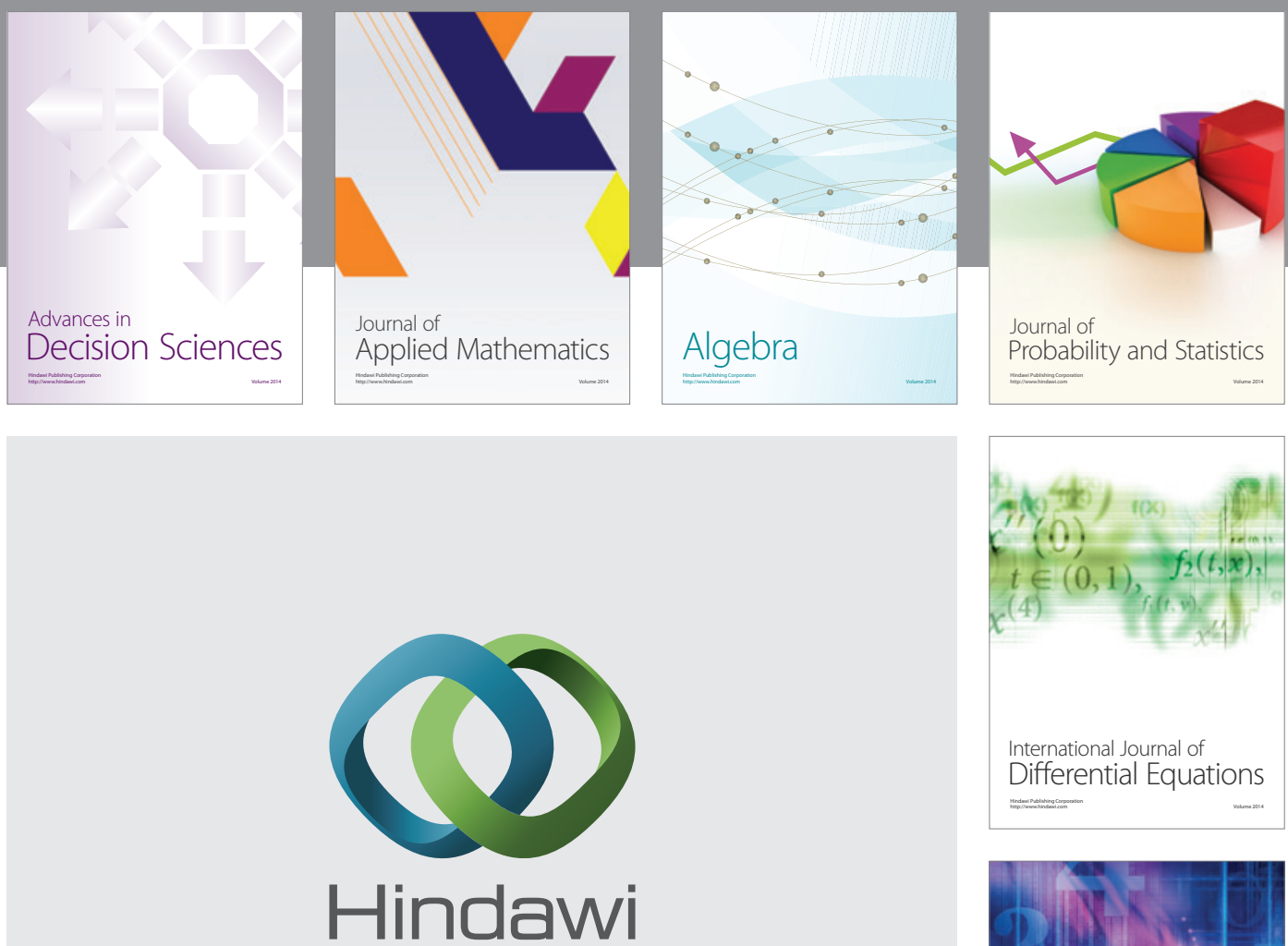

Submit your manuscripts at http://www.hindawi.com
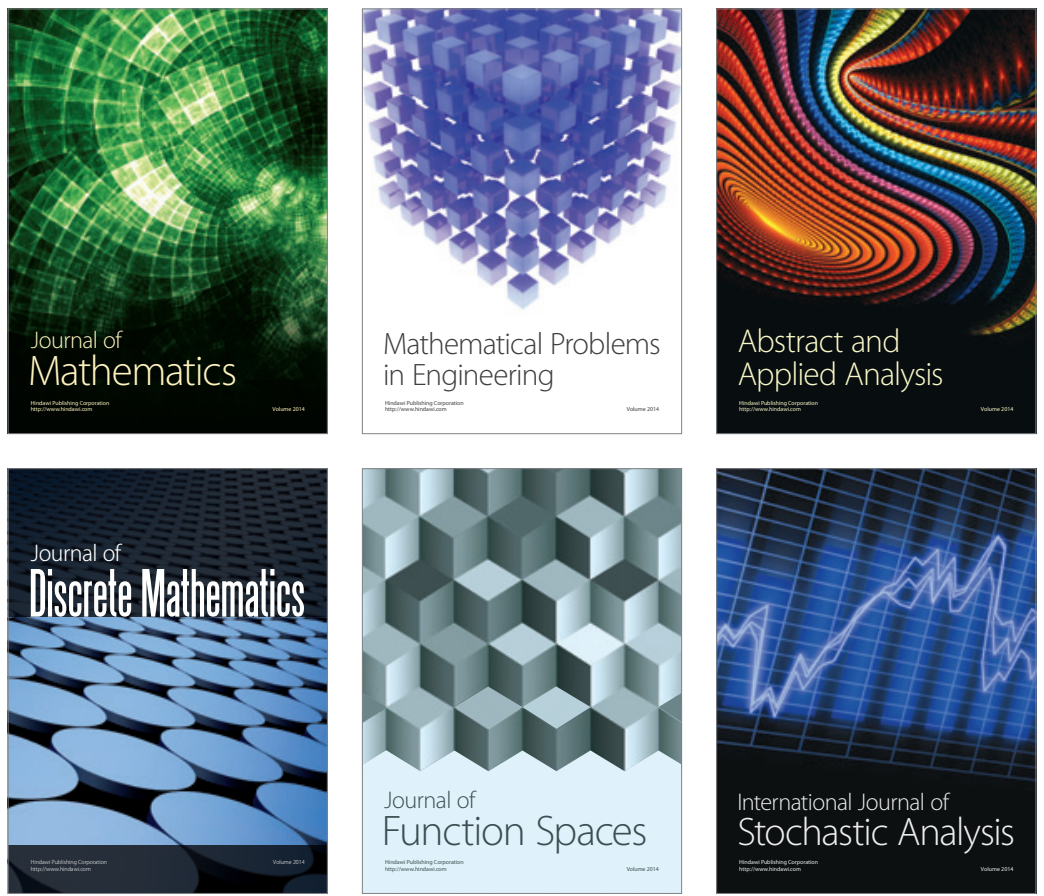

Journal of

Function Spaces

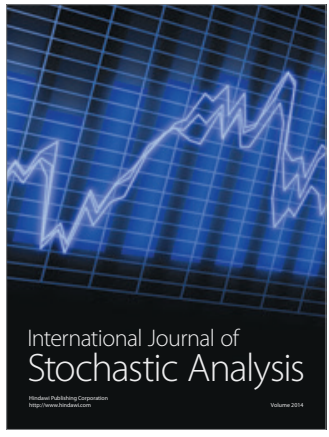

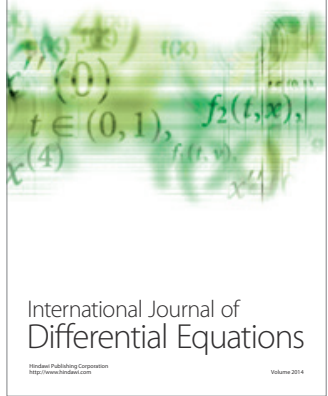
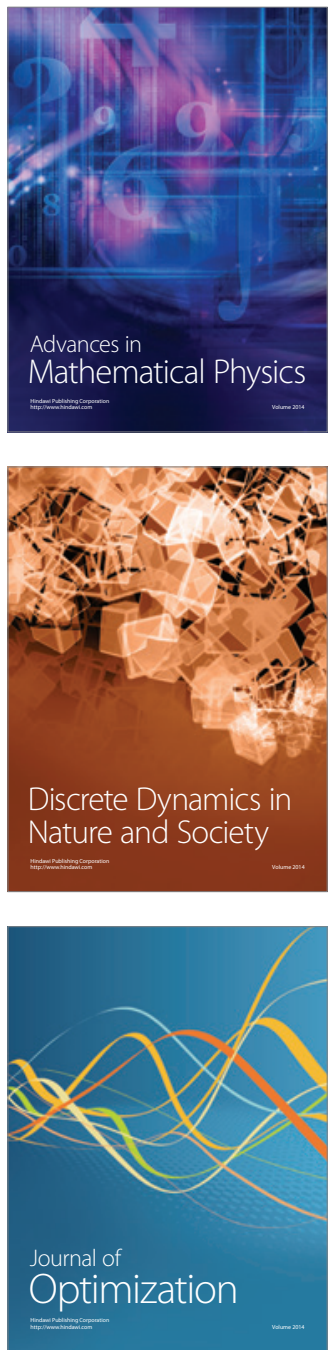